\title{
Measurement Method for the Assessment of the Energy Consumption of Cooking Setups
}

\begin{abstract}
Energy efficiency in household appliances is a concern in energy saving scenarios. Especially, the equipment for cooking is very likely for energy waste since a lot of energy is used to produce steam, where simmering the goods to be cooked is sufficient. The reduction of this loss is the main goal of the energy optimization of cooking equipment. In standardization, only the measurement of the energy consumption of the heat up of water is regulated, not the cooking procedure itself, since the standard is dedicated to assess the heat transfer from the cooking zone to the water in a pan. However, it is the cooking phase after heat up that accounts for the largest part of energy waste. Thus, a comparison of the energy consumption between different cooking setups is difficult. In the work presented, a measurement procedure has been worked out to measure the energy needed for a cooking cycle with a cooking setup, which consists of the cooking zone, the cookware, and the regulating equipment, as well as to determine the influence of the behaviour of the user.
\end{abstract}

KEYWORDS: home appliances, energy conservation, measurements

\section{Introduction}

Energy conservation has gained significant importance over the last years. Also, household appliances are increasingly becoming interesting for energy conservation, where, in the cooking process, considerable amounts of energy can be saved because in boiling, usually most energy is used to produce steam rather than for cooking the goods intended to be cooked. This leads to a considerable waste of energy because at the boiling temperature of water, the energy consumption goes into the increase of entropy without increasing the temperature of the water. Since the specific heat capacity $c_{p}$ of water is $\sim 4.2 \mathrm{~J} / \mathrm{g} \cdot \mathrm{K}$ and the specific enthalpy of vaporization $\Delta H$ is $2257 \mathrm{~J} / \mathrm{g}$ [1], $\sim 7$ times the energy is needed to evaporate $1 \mathrm{~g}$ of water than heating it from 20 to $100^{\circ} \mathrm{C}$. Thus, the use of cooking appliances that heat the goods to $100^{\circ} \mathrm{C}$ by transforming as little water as possible into steam can lead to the conservation of huge amounts of energy. Principally, the effect of simmering the goods by minimizing the energy loss due to evaporation of the water can be achieved manually by heating up the water with the maximum power of the heating device and reducing the heat in the appropriate moment. This is quite difficult because of the heat storing capabilities of the cooking zones as well as of the cookware. Thus, the manual cooking generally leads to excessive energy losses. To overcome this waste, energy saving cooking devices are developed, which use various technologies to regulate the power of the cooking zone in the sense that the water is kept as close as possible to $100^{\circ} \mathrm{C}$ by avoiding the boiling of the water, not to forget the use of insulated cookware, which is already available for many years.

In manual cooking, the procedure can be divided into two phases: First, the heat up phase (phase 1) where the water is heated up until vapor occurs. Then, the actual cooking phase follows (phase 2), where the goods are kept on the temperature necessary to

Manuscript received January 26, 2010; accepted for publication September 26, 2010; published online October 2010.

${ }^{1}$ Scientist, Swiss Federal Laboratories for Materials Testing and Research (EMPA), 8600 Dübendorf, Switzerland.

${ }^{2}$ Lab Assistant, Swiss Federal Laboratories for Materials Testing and Research (EMPA), 8600 Dübendorf, Switzerland. maintain the chemical processes until the goods are ready for consumption. In automatic cooking, phase 1 does not necessarily occur as clear as in manual cooking since the heating up and the simmering merge into one.

Presently, no standard exists to compare the performance of automatic cooking setups or to manual cooking including phase 2 . In IEC 60350, the energy consumption to heat up a given amount of water for $75^{\circ} \mathrm{C}$ from 15 to $90^{\circ} \mathrm{C}$ is measured, and the energy needed for the heat transfer from the cooking zone to the water in a pan is assessed [2]. While this method is suitable to characterize the energy transfer from the cooking zone to a standardized cookware in phase 1 , it does not account for the capabilities of the cookware to minimize the energy consumption in phase 2 . However, it is phase 2 where new cooking setups aim to save energy, be it as regulated cooking zones or as cookware that actively regulates the cooking zone. It was the goal of this work to establish a measurement procedure that allows the measurement of the energy consumption in both cooking phases and to compare the energy consumption of different cooking setups, which consist of the cooking zone, the cookware, the regulating equipment, and the behaviour of the user.

\section{Measurement Procedure}

\section{Course of Actions}

Since in IEC 60350 the heating up of water is used to assess the energy heat transfer, it was decided to use the cooking operation with water to characterize the energy consumption of a cooking cycle. As cooking goods, we chose potatoes for the measurements, with the time above a given minimum temperature in the potatoes as the measured parameter. First, dummy cooking goods have been defined, which can repeatedly be used and mimic the behaviour of a potato. Second, a decision parameter has been defined, when energy is usually reduced in manual cooking. Third, in manual cooking experiments and from literature, the temperature necessary and the time required for the core of a potato to be cooked have been 


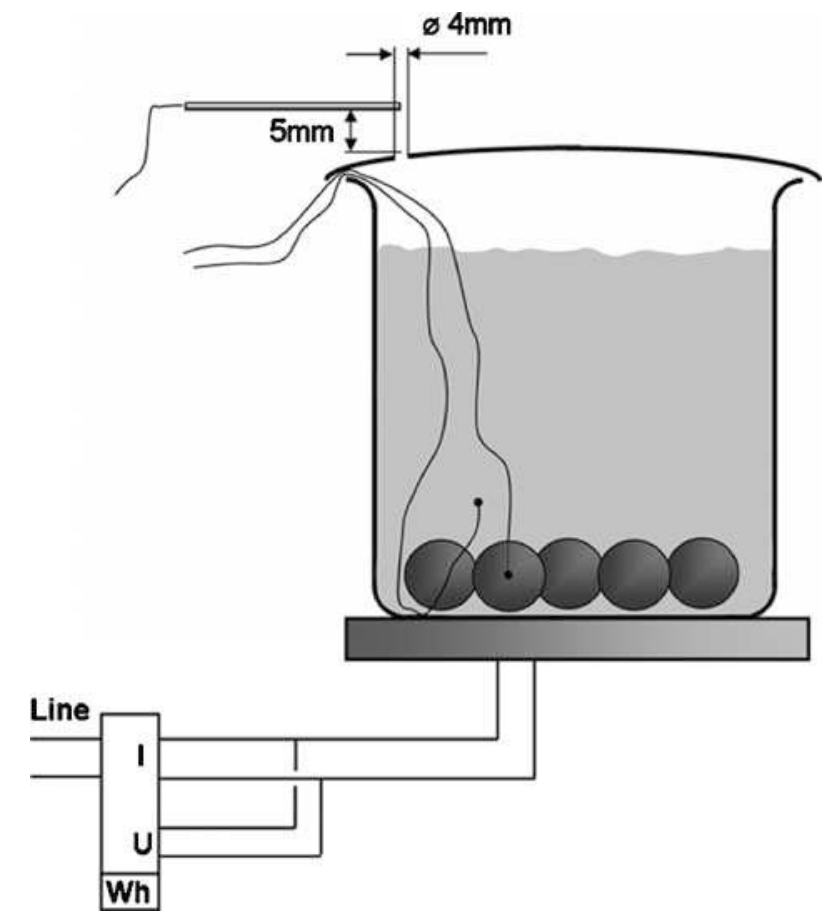

FIG. 1-Sketch of the measurement setup for energy consumption. One thermal element is used for the detection of vapor, one for the measurement of the temperature in the dummy cooking goods and one to measure the temperature of the water. Because the cooking zone can be regarded to as an Ohmic heater, the energy could be determined by a continuous integration of the effective energy by measuring the current and the voltage drop.

specified, and a measurement procedure to determine the energy consumption of the cooking operation has been established.

In the end, the procedure has been applied to a measurement example to demonstrate its suitability.

\section{Measurement Setup}

The measurements where done in a cooking pot made of steel with a diameter of $220 \mathrm{~mm}$ and with a lid. A second lid, equipped with a measurement device and a radio control unit to control the heat generating device, has been used for the automatic cooking operation in the measurement example. The heat generating device was a stand alone, single plate glass ceramic radiant cooking zone with a diameter of $210 \mathrm{~mm}$ equipped with a heat limiter and a manual power setting possibility and with an arbitrary scale from 1 to 9 ; it was equipped with a device to communicate with the cookware if desired. The same setup was used in all measurements. The maximum power of the cooking zone has been measured to be $1.6 \mathrm{~kW}$. In the pot, five dummy potatoes where placed, one of them equipped with a K-type thermal element in the center. In the lid of the pot, a hole of a diameter of $4 \mathrm{~mm}$ has been drilled, and another type $\mathrm{K}$ thermal element mounted in an aluminum tube with a diameter of 3 $\mathrm{mm}$ has been placed above the hole in the lid as steam indicator. A third K-type thermal element has been placed in the water fixed with a weight and with the measurement point $\sim 1 \mathrm{~cm}$ above the dummies. The energy consumption of the cooking zone has been monitored with an electronic power meter (Fig. 1). During the cooking cycles, the temperatures of the steam indicator $\vartheta s$, the water $\vartheta w$, and inside the dummy $\vartheta d$ as well as the energy consumption where recorded.

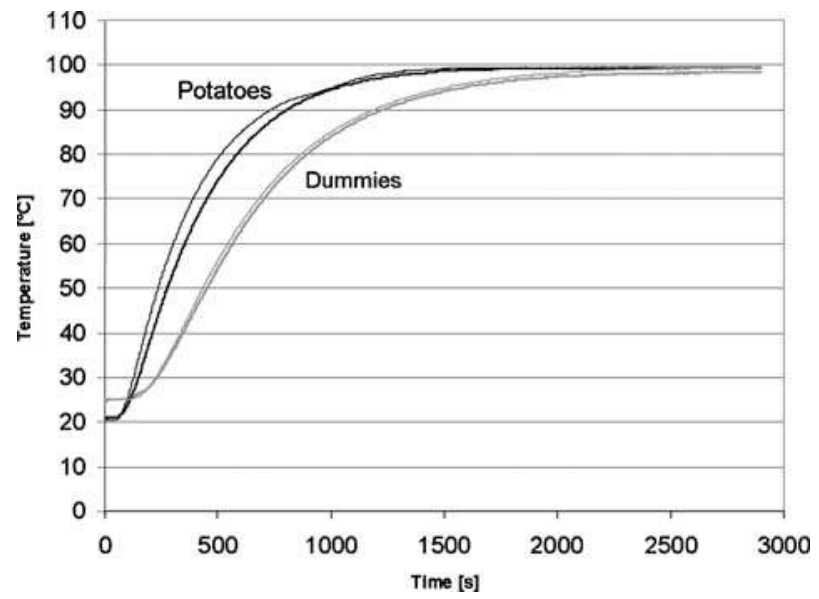

FIG. 2-Comparative measurement of the temperature in two silicon balls and two potatoes of similar size.

For each measurement, the pot has been refilled with 21 of fresh water with a temperature of $21^{\circ} \mathrm{C} \pm 2.5^{\circ} \mathrm{C}$. The time between the measurements was $15-30 \mathrm{~min}$.

\section{Dummy Potatoes}

To mimic the cooking of potatoes, silicon balls $40 \mathrm{~mm}$ in diameter and weighting $36 \mathrm{~g}$ each have been used. A hole of $1.5 \mathrm{~mm}$ diameter and $20 \mathrm{~mm}$ depth has been drilled into a ball, and a type $\mathrm{K}$ thermal element has been placed in the center of the ball. The initial measurements of the internal temperature of silicon balls compared with that of potatoes of similar size, with a laboratory heating plate with a size of $200 \times 280 \mathrm{~mm}^{2}$, showed a reasonable agreement of the heat up of the dummies and the potatoes (Fig. 2). However, during the measurements, it became clear that a thorough sealing of the drilled hole in the dummies is necessary in order to avoid hot water penetrating into the measurement point of the $\mathrm{K}$ thermal element.

\section{Defining the Occurrence of Vapor}

As the decision indicator to reduce the energy input in manual cooking or to remove the cookware from the cooking zone, the occurrence of steam has been used since a quick survey amongst housewives by the author showed that phase 1 is considered to be terminated as soon as vapor is visibly emitted between the pot and the lid. In order to detect the occurrence of vapor reproducibly, a type $\mathrm{K}$ thermal element has been placed above a hole in the lid as steam indicator. In the course of the measurements, a distance of 5 $\mathrm{mm}$ above a hole with a diameter of $4 \mathrm{~mm}$ has been shown to give good results. The visible formation of steam has been defined at the point where the steam indicator showed a temperature $\vartheta s=95^{\circ} \mathrm{C}$ (Fig. 3).

\section{Definitions of Cooking Parameters}

In starch containing goods, such as potatoes, the denaturation of starch such as amylose and amylopectin principally defines the cooking. The minimum temperature for this process is $60^{\circ} \mathrm{C}$ [3]. However, potatoes also contain tuberin, a simple protein that needs to be heated to $80^{\circ} \mathrm{C}$ to coagulate [4]. It has been shown that sweet potatoes need to be heated to $80^{\circ} \mathrm{C}$ to be agreeably edible [5]. In 


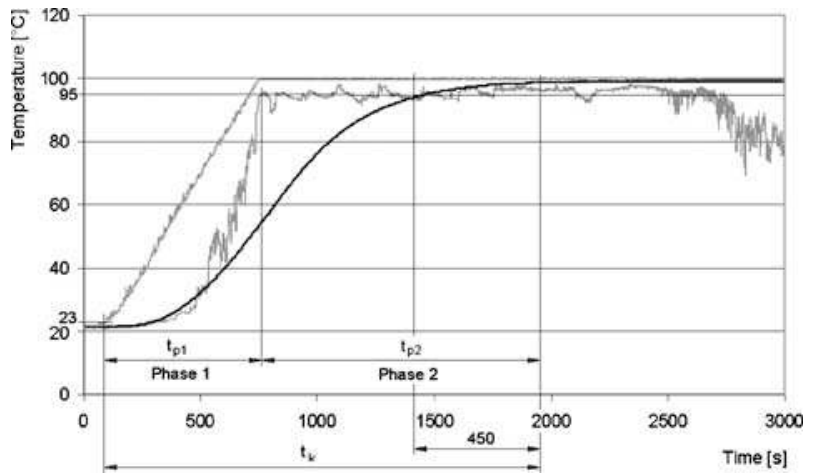

FIG. 3-Definition of cooking parameters.

Ref 4, it has been found that the core of potatoes at doneness reached $92-98^{\circ} \mathrm{C}$ if an acceptable culinary quality is to be obtained. In Refs 6-8, is stated that potatoes need to be cooked for 15-45 min after the water boils. For this investigation, $20 \mathrm{~min}$ after the water boils have been chosen as cooking time.

In the measurements of manual cooking, it has been seen that $\sim 20 \mathrm{~min}$ after the steam indicator showed $\vartheta s=95^{\circ} \mathrm{C}$, the core of the dummy potatoes surpassed $95^{\circ} \mathrm{C}$ for $450 \mathrm{~s}$. Thus, phase 2 with the time $t_{p 2}$ has been defined as the phase after $\vartheta s \geq 95^{\circ} \mathrm{C}$ until the core of the dummy surpassed $95^{\circ} \mathrm{C}$ for $450 \mathrm{~s}$, and the total cooking time $t_{k}$ has been defined as the time from $t=0$ until $\vartheta d$ in the center of the potato surpassed $95^{\circ} \mathrm{C}$ for $450 \mathrm{~s}$, which corresponds to the sum of the time spans needed to complete phase 1 and phase 2 $\left(t_{p 1}+t_{p 2}\right)$.

In order to have a defined starting point for the measurements, the time has been set to 0 when the temperature of the water reached $23^{\circ} \mathrm{C}$. For the assessment of the energy consumption for $t_{p 1}$ and $t_{k}$, the times for phase 1 and phase 2 , respectively, have been used (Fig. 3).

\section{Energy Measurement}

The energy used for the entire cooking procedure has been measured with a three-phase power metre with an integrated energy integration routine. The sampling rate for the integration was $0.55 \mathrm{~s}$, and the energy was manually recorded at various intervals. The energy consumption for $t_{p 1}$ and $t_{k}$ has been extracted from the energytime graph by interpolation of the recorded data (Fig. 4).

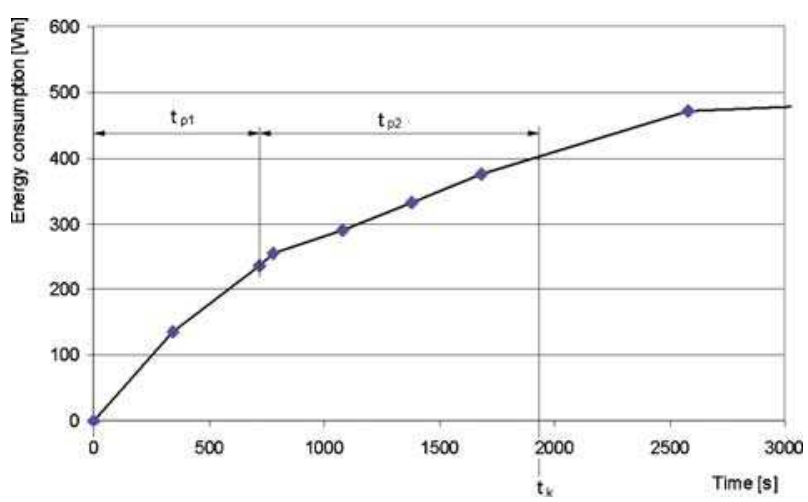

FIG. 4-Determination of energy consumption.

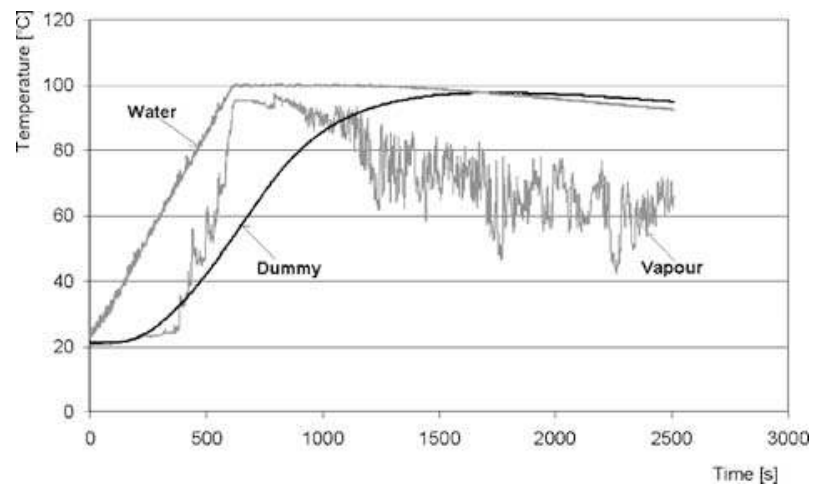

FIG. 5-Temperatures in measurement 1.

\section{Measurement Example}

\section{Goal of the Measurement}

The measurement procedure outlined has been used to compare the energy consumption of an electronically regulated cooking pot to manual cooking. The automatic device determines if the water in the pot starts to boil and transfers this data to the cooking zone, which reduces the energy flow in order to keep the temperature of the water as close as possible to $100^{\circ} \mathrm{C}$ without wasting energy due to excessive evaporation. The cooking zone was the same electrical glass ceramic cooking zone as outlined previously with the automatic mode activated.

\section{Manual Operation}

In manual operation, the power of the cooking zone has been set to level 9 in phase 1 and set to levels 1, 6, and 9, respectively, for phase 2. During the measurements it, became apparent that the cooking zone had a security switch, which disabled overheating by reducing the energy drastically if the plate became excessively hot.

In measurement 1 , the power of the cooking plate was manually reduced to level 1 as soon as the steam indicator showed $95^{\circ} \mathrm{C}$ which was $\sim 620 \mathrm{~s}$ after the start of the test. The silicon balls then had a temperature of $54^{\circ} \mathrm{C}$. After reducing the power, the temperature of the steam indicator dropped. However, the temperature of the water stayed above $99^{\circ} \mathrm{C}$ for another $800 \mathrm{~s}$. The silicon balls reached $95^{\circ} \mathrm{C}$ after 23 min from the beginning (Fig. 5), which

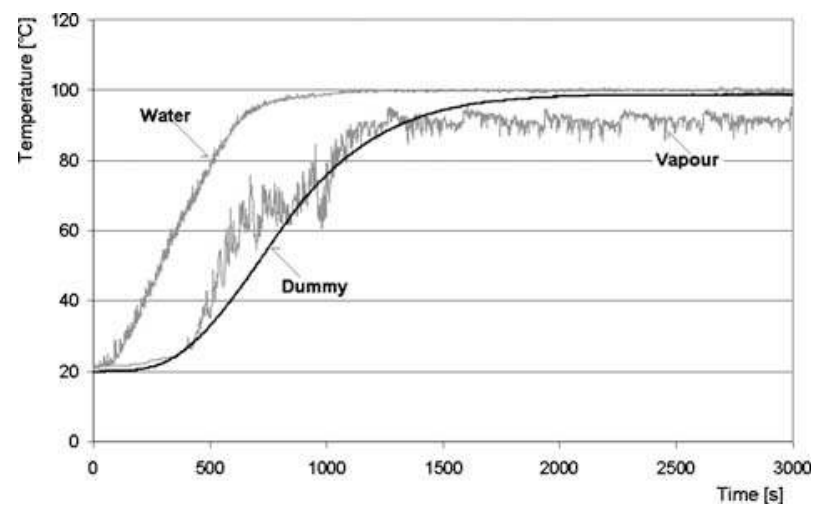

FIG. 6-Temperatures of automatic operation. 
TABLE 1-Summary of the measurements.

\begin{tabular}{lcccccc}
\hline & & \multicolumn{2}{c}{ Time (s) } & & \multicolumn{2}{c}{ Consumption (W·h) } \\
\cline { 3 - 4 } Meas. & Power Level, $\vartheta s>95^{\circ} \mathrm{C}$ & $t_{p 1}$ & $t_{k}$ & & $t_{p 1}$ & $t_{k}$ \\
\hline 1 & 1, manual & 623 & 1800 & & 199 & 258 \\
2 & 6, manual & 678 & 1835 & & 230 & 361 \\
3 & 9, manual & 676 & 1834 & & 230 & 514 \\
4 & Auto & & 1896 & & 263 \\
\hline
\end{tabular}

added to 30 min cooking time together with the $450 \mathrm{~s}$ above $95^{\circ} \mathrm{C}$ necessary to cook a potato.

The power consumption for phase 1 was $200 \mathrm{~W} \cdot \mathrm{h}$. An additional $58 \mathrm{~W} \cdot \mathrm{h}$ was consumed in phase 2 , summing up to $258 \mathrm{~W} \cdot \mathrm{h}$ in $t_{k}=30 \mathrm{~min}$.

In measurement 2, the power was reduced to level 6 after phase 1 , and the total energy consumption was $361 \mathrm{~W} \cdot \mathrm{h}$.

In measurement 3 , the power level was not reduced manually after phase 1 . In this test, the power was reduced by the cooking zone after $\sim 2000 \mathrm{~s}$, which was after $t_{k}$, however. This resulted in a total energy consumption of $514 \mathrm{~W} \cdot \mathrm{h}$.

\section{Automatic Operation}

In automatic operation, phase 1 was not visible with $\vartheta s$ since the regulating system avoids the energy wasting process of producing steam. An increase of the temperature of the vapor emanating from the hole in the lid could be observed, but $\vartheta s$ never reached $95^{\circ} \mathrm{C}$ and no steam was visible during the cooking operation. However, this was meaningless since the occurrence of vapor did not have any significance. In the temperature-time graph, the regulating of the device is well visible in the sense that the water temperature did not rise linear until $100^{\circ} \mathrm{C}$ and then suddenly stop rising but slowly approached $100^{\circ} \mathrm{C}$ (Fig. 6). The dummy reached $95^{\circ} \mathrm{C}$ after 26 min; the energy consumption at $t_{k}$ was $263 \mathrm{~W} \cdot \mathrm{h}$.

Table 1 gives a summary of the results. The energy consumptions have been extracted from the energy-time graphs as outlined previously.

\section{Summary}

The measurement setup presented provides an easy method to compare the energy consumption of a cooking setup with water. The method allows the comparison of the energy consumption of automatic cooking devices as well as cooking setups used in manual cooking such as insulated cookware. As a decision parameter for the completion of the cooking procedure, a minimal time above a necessary temperature in the center of reusable test bodies has been defined. Thus, the measurement involves a temperature measurement device in the center of a test-body and a power metre with an integration routine. The energy consumption of a cooking procedure can be measured by integrating the energy consumption from the beginning of the test until the temperature in the center of the test-body exceeds the defined minimal time. In the investigations, it was found that potatoes need to be kept above $95^{\circ} \mathrm{C}$ for a minimum of $450 \mathrm{~s}$. These parameters have been chosen for the example measurement to have a connection to a real application but can be set to any combination of values that need to be kept constant for comparative tests. As the decision indicator to reduce the power in manual cooking, other indicators such as noise, formation of bubbles, or a threshold temperature could be used principally. However, the occurrence of steam seems to be a common sign for users that the water reached the desired temperature.

By the measurement setup, the energy consumption of an entire cooking setup, consisting of the cooking zone, the cookware, and possible regulating equipment as well as the behaviour of a user, is measured for not only the efficiency of a single element of the setup. This means that the influence of an insulation of cookware or the configuration of its base on the energy consumption, the energy needs of steam cookers, or the efficiency of dedicated cooking zone-cookware combinations can be compared. It also means that the influence of different cooking behaviours of users can be quantified. The measurement setup presented is suited to compare the energy consumption of cooking procedures in boiling. While the method is not applicable for grilling, it can be potentially used for steaming because also in this case, a given time above a given temperature in the dummies can be taken as a method to determine the cooking procedure and thus to compare the energy consumption of steaming setups.

Of course, to compare varying cooking setups, it is necessary to define a standard setup where all the influencing parameters are standardized. However, once this is done, the measurement method can be used to compare variations of any of the parameters to the standard setup.

The measurement example showed that the method is well suited to compare manual cooking with an automatic cooking device and also to compare the effect of variations in the cooking behaviour of the user.

The measurement of the energy consumption in phase 1 shows a variation in the measurements of roughly $\pm 5 \%$, although it should be constant and given by the heat capacity of the cookware, the water, and the cooking zone as well as the heat transfer from the heater to the cookware. Possible reasons for these variations are the placement of the cookware on the cooking zone, the time between the measurements to let the heating zone cool down, and a certain heating up of the water upon refilling due to the heat storage capabilities of the cookware, and the determination of the energy consumption by interpolation. Another factor, which has not been accounted for in the sample measurement, is the air pressure, which influences the maximum temperature of the water and thus the heat transfer to the goods. However, since the investigations have been done within 1 day, this factor should not have influenced the measurements.

\section{References}

[1] Lide, D. R., Handbook of Chemistry and Physics, CRC Press, Boston, MA, 1992.

[2] IEC 60350, 2005, "Electric Cooking Ranges, Hobs, Ovens 
and Grills for Household Use-Methods for Measuring Performance," International Standard, 2.2nd ed.

[3] Barham, P., The Science of Cooking, Springer, Berlin, Heidelberg, New York, 2002.

[4] Sweetman, M. D., "The Physico-Chemical Changes Produced by the Cooking of Potatoes," Am. Potato J., Vol. 10, No. 9, 1933, pp. 169-173.

[5] Sinoda, O., and Kodera, S., "On the Critical Temperature in Cooking the Sweet Potato," Biochem. J., Vol. 26, No. 3, 1932, pp. 650-657.

[6] Tecstra Systems, Cooking Tips \& Advice, http://www. recipetips.com/kitchen-tips/t--826/all-about-potatoes.asp (Last accessed September 2010).

[7] Oetker, A., Vegetarisches Kochbuch [Vegetarian Cookbook], Ceres, Bielefeld, Germany, 1994, p. 70.

[8] Zäch, R., 1931, Die Neuzeitliche Küche[The Modern Kitchen], Emil Frey Verlag, Zürich, Switzerland, p. 144. 